\title{
Two-year clinical outcomes of radiofrequency focal ablation using a navigable plasma disc decompression device in patients with lumbar disc herniation: efficacy and complications
}

This article was published in the following Dove Press journal: Journal of Pain Research

\section{Nack Hwan Kim' \\ Youngki Hong ${ }^{2}$ \\ Sang-Heon Lee ${ }^{3}$}

'Biomedical Research Center, Korea University Anam Hospital, Seoul, Republic of Korea; ${ }^{2}$ Department of Sports Medicine, Cheongju University, Cheongju, Republic of Korea; ${ }^{3}$ Department of Physical Medicine and Rehabilitation, Korea University Anam Hospital, Seoul, Republic of Korea
Correspondence: Sang-Heon Lee Spine Center, Korea University Anam Hospital, Anam-dong 5-ga, Seongbuk-gu, Seoul I36-70I, Republic of Korea

Tel +8229206471

Fax +82 29299951

Email spinelee@gmail.com
Purpose: To report on the 2-year clinical outcomes of focal ablation using a navigable plasma disc decompression device in patients with lumbar herniated nucleus pulposus (HNP).

Patients and methods: This was a prospective, single-cohort study conducted in a clinical center. A total of 170 patients with lumbar HNP were assessed for pain intensity using the visual analog scale (VAS), for disability level using the Oswestry Disability Questionnaire (ODI), for health-related quality of life using the short form-36 version 2 of the bodily pain scale (SF$36 \mathrm{BP}$ ), and for the angles of passive straight leg raise (SLR) test. The herniated portions of the target discs were ablated using a navigable catheter under a well-instructed protocol with informed consent. Outcome data were prospectively collected before the procedure: 1 week after the procedure: and then 1, 3, 6, 12, and 24 months postoperatively. For statistical analysis, repeated-measures analysis of variance was performed.

Results: Two years after the procedure, the mean VAS decreased from $7.1 \pm 1.7$ to $2.1 \pm 1.9$, the mean ODI decreased from $50.9 \pm 17.2$ to $20.3 \% \pm 14.6 \%$, and the mean SF-36 BP increased from $38.8 \pm 8.4$ to $45.8 \pm 9.4(P<0.05)$. On the SLR test, the angular change after 2 years improved considerably from $51.2 \pm 17.3$ to $85.0 \pm 9.3$ degrees. There was $90 \%-100 \%$ VAS improvement in $25.9 \%$ and $50 \%-90 \%$ VAS improvement in $52.4 \%$ of the patients; $1.8 \%$ experienced pain aggravation compared with the initial VAS. Two subjects showed short-term foot drop, whereas one subject showed the severe complication of foot drop for more than 6 months. The recurrence rate ranged from $4.7 \%$ to $11.5 \%$.

Conclusion: These findings suggest that focal ablation of herniated discs using a navigable disc decompression device is worth considering for relieving pain related to lumbar HNP. However, the development of more advanced technologies and methods for safety and efficiency is necessary. Keywords: low back pain, herniated disc, minimally invasive surgical procedures, percutaneous catheter ablation

\section{Introduction}

Low back and lower extremity pain are the most common presenting symptoms of lumbosacral herniated nucleus pulposus (HNP), and they can be attributed to torn annulus inflammation and neural impingement of herniated disc tissue.

Open discectomy is recommended if conservative treatment fails, and the success rate is reported to be $70 \%-90 \% .^{1,2}$ Indications for surgery include moderate or severe neurological damage that is clinically confirmed, unclear response to the oral analgesics, or a high risk of relapse with no other available therapeutic options. ${ }^{3}$ Aside from the 
absolute indications for surgery, deciding whether surgery is recommended involves consideration of the empirical elements on the part of the physician and therefore requires close discussion with the patient. For this reason, the selection of surgical methods in a clinical setting is quite limited. Although there is scarce precise evidence regarding the rejection of spinal surgery, given the cancelation of a general elective surgery, there is a high incidence of rejection due to failure of a prior lumbar spine surgery, fear of the surgery itself, inability to undergo general anesthesia because of underlying diseases, and surgery postponement because of a patient's work commitments. ${ }^{4}$

Many minimally invasive spine intervention methods and devices are being developed to maximize the surgical advantages and minimize its disadvantages. Our research team has developed a radiofrequency catheterization method and previously investigated its clinical results. ${ }^{5}$ A navigable plasma disc decompression catheter was designed for localized access and selective treatment of HNP using a radiofrequency coblation technique. We have previously reported on the clinical efficacy of catheters in patients with lumbar intervertebral disc herniations related to radicular pain using a navigable catheter capable of selectively reaching the target point in the disc along with safe and localized tissue removal through coblation $(n=27){ }^{6}$

The aim of the present study was to report the 2-year follow-up data after minimally invasive spinal interventions using a navigable plasma disc decompression catheter. We observed clinical symptoms and adverse events after intervention through clinical assessment tools and patient interviews.

\section{Materials and methods}

\section{Subjects}

Our institutional ethics committee (Korea University Anam Hospital, Republic of Korea) approved the study, and all patients provided written informed consent. The subjects were informed of the details of all procedures and patient protection regulations, and we emphasized their voluntary participation and freedom to withdraw. One physician performed all the intradiscal catheterizations, while other physicians collected the data and conducted the analyses.

From March 2010 to April 2014, lower back and lower extremity pain patients at our hospital were diagnosed with lumbosacral HNP with radiating pain by combining magnetic resonance image (MRI) and physical examination findings, especially the positive straight leg raise (SLR) test. ${ }^{7,8}$
All the patients visiting our clinical center had been referred by the primary clinic. Patients who received conservative management for more than 3 months and were recommended for surgery by the primary physician were informed about the study. Since the exact type, amount, and quality of conservative treatment were unknown to us, we administered our own conservative treatment for 1 month including physical therapy, oral analgesics, and epidural steroid injections, after which we once again recommended our procedure to the patients.

The exclusion criteria were as follows: clinically significant lumbar HNP of two or more levels on MRI, severe degeneration of the target disc on MRI (Grade V using the Pfirrmann grading system),${ }^{9}$ significant thoracolumbar signal change in the spinal cord on MRI, any signs of upper motor neuron lesion upon physical examination, symptom-related lumbosacral bony malformation, prior lumbar spinal surgery history, symptom-related psychological disorders (major depression disorder, somatization disorder), hematologic disorders, or chronic painful musculoskeletal conditions (fibromyalgia, myopathy, polyneuropathy, small fiber neuropathy).

\section{Device, patient preparation, and procedure protocols}

The principal device was a navigable plasma disc decompression catheter called L'DISQ (U \& I Corporation, Uijeongbu-si, Gyeonggi-do, Korea), which can move through the intradiscal course to the focal herniated tissue with considerable accuracy and ablate part of the tissue for selective decompression by high-energy plasma particles. ${ }^{5}$

The subject was in a prone position after being equipped with a proper monitoring device. A 25-gauge spinal needle was first placed into the "safety triangle" of the ipsilateral foramen at the target disc, and contrast dye was injected to outline the herniated disc. ${ }^{10}$ This spinal needle provided the saline needed for coblation and tissue cooling. Next, the catheter entry point was marked on the skin $12-15 \mathrm{~cm}$ contralateral to the midline. The endplates of the target disc were aligned on fluoroscopic guidance, and the fluoroscopic target point was located at the approximate dorsal one-third of the disc on the 15-degrees fluoroscopic view from a zerodegree lateral projection. After anesthetizing the skin and subcutaneous fascia to the superior articular process, the introducer needle was directed gently toward the annular surface of the contralateral foramen on fluoroscopic guidance. Prior to advancing the introducer needle into the disc, the anteroposterior and lateral projection was checked. The 
final position of the needle tip must be placed at the adjacent intradiscal area of the herniated portion (Figure 1).

After confirming the introducer needle position with the lateral and AP views, the stylet was removed and the catheter wand was advanced through the introducer needle to the center of the herniated portion using fluoroscopic monitoring of the AP and lateral views. Before ablation, negative motor nerve stimulation confirmed that the needle was not close to the traversing or exiting nerve root. During the ablation, the tip of the wand was continuously rotated and moved back and forth to increase the ablated volume. During ablation, normal saline was injected at about $0.1 \mathrm{~mL}$ per second through the ipsilateral spinal needle. When the portion was decompressed, the resistance of wand tip motion was reduced. The 5-second ablation was completed 40-60 times.

\section{Outcome measures}

Assessments were conducted using the visual analog scale (VAS), the Oswestry Disability Index (ODI), and the short form-36 version 2 of the bodily pain scale (SF-36 BP). The VAS rates pain severity as a score from 0 to 10 . The ODI assesses low back pain-related disability: the higher the score, the more severe the disability. The bodily pain scale is a patient-reported subscale of the SF-36 BP that measures health status; specifically, it asks patients about any limitations in daily life due to pain, and the lower the score, the greater the disability. We collected initial data 1 or 2 weeks before the procedure and scheduled follow-up visits for 1, 4, and 12 weeks, 6 months, and 1 and 2 years after the procedure. During the individual interviews, we collected information from the patients regarding side effects, discomfort, and related medical history. At the last visit, the perceived change in leg and back pain was surveyed by 5-point Likert scale (pain-free, much better, somewhat better, unchanged, worse).

To identify the ablated portion of the disc, evaluate the catheter pathway and the contour of the posterior spinal

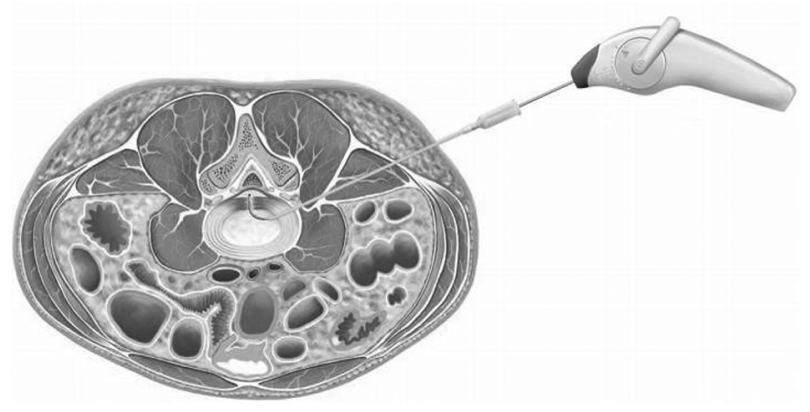

Figure I Schema of the placement of device in disc with central herniation. column, and check the distribution of byproduct gas and other visible complications, we took computed tomographic (CT) images immediately postoperatively that were interpreted by a special radiologist.

We conducted repeated-measures ANOVA to analyze the clinical findings before the procedure and the outcomes at each follow-up, and we compared the periodic outcomes using paired $t$-tests at 0.05 significance. The correlation of VAS and perceived symptom changes of back and leg pain were analyzed through Spearman's rank test. We used SPSS 22.0 for Windows (IBM Corporation, Armonk, NY, USA).

\section{Results \\ Subjects}

During the study period, 379 subjects with relevant symptoms visited our center. All of the subjects had been informed about the new procedure, but 160 were excluded based on past medical history, image findings, or electrophysiologic test results. Of the remaining 219 patients initially enrolled, 27 refused the procedure and 13 underwent the procedure but dropped out during follow-up (Figure 2).

For the final analysis, we collected data from 170 subjects (98 males and 72 females) with a mean age of 42.9 years with a range of 20-79 years. The mean symptom duration was 16.4 months with a range of 6-72 months. The targeted discs were L5/S1 in 72 patients, L4/5 in 86, L3/4 in ten, $\mathrm{L} 2 / 3$ in one, and L1/2 in one. The grade of disc degeneration and type of herniation were investigated (Table 1). ${ }^{9,11}$ As an example of procedure, MRIs and post-operative CT images are shown in Figure 3.

\section{Outcomes}

Compared with the preoperative baselines, the VAS, degree of the SLR test, ODI, and SF-36 BP scores showed statistical improvement 1 week postoperatively, gradual improvement until 6 months, and sustained improvement after 2 years $(P<0.05$; Figure 4$)$. The average VAS score was 7.1 \pm 1.7 before the procedure, 3.3 \pm 2.01 -week postoperatively, and $2.1 \pm 1.9$ after 2 years. The SLR test findings, which we measured at intervals of 10 degrees using a goniometer, reflected significant improvement, from $51.2 \pm 17.3$ to $77.8 \pm 14.2$ at 1 -week postoperatively. The mean ODI score showed a significant decrease at 1-week postoperatively, a gradual decrease at 6 months, and improvement from 50.9 \pm 17.2 20.3 \pm 14.6 after 2 years. The SF-36 BP scores increased significantly at 1 -week postoperatively compared with the baseline of $38.8 \pm 8.4$; the scores continued to improve steadily for 2 years to $45.8 \pm 9.4$. 


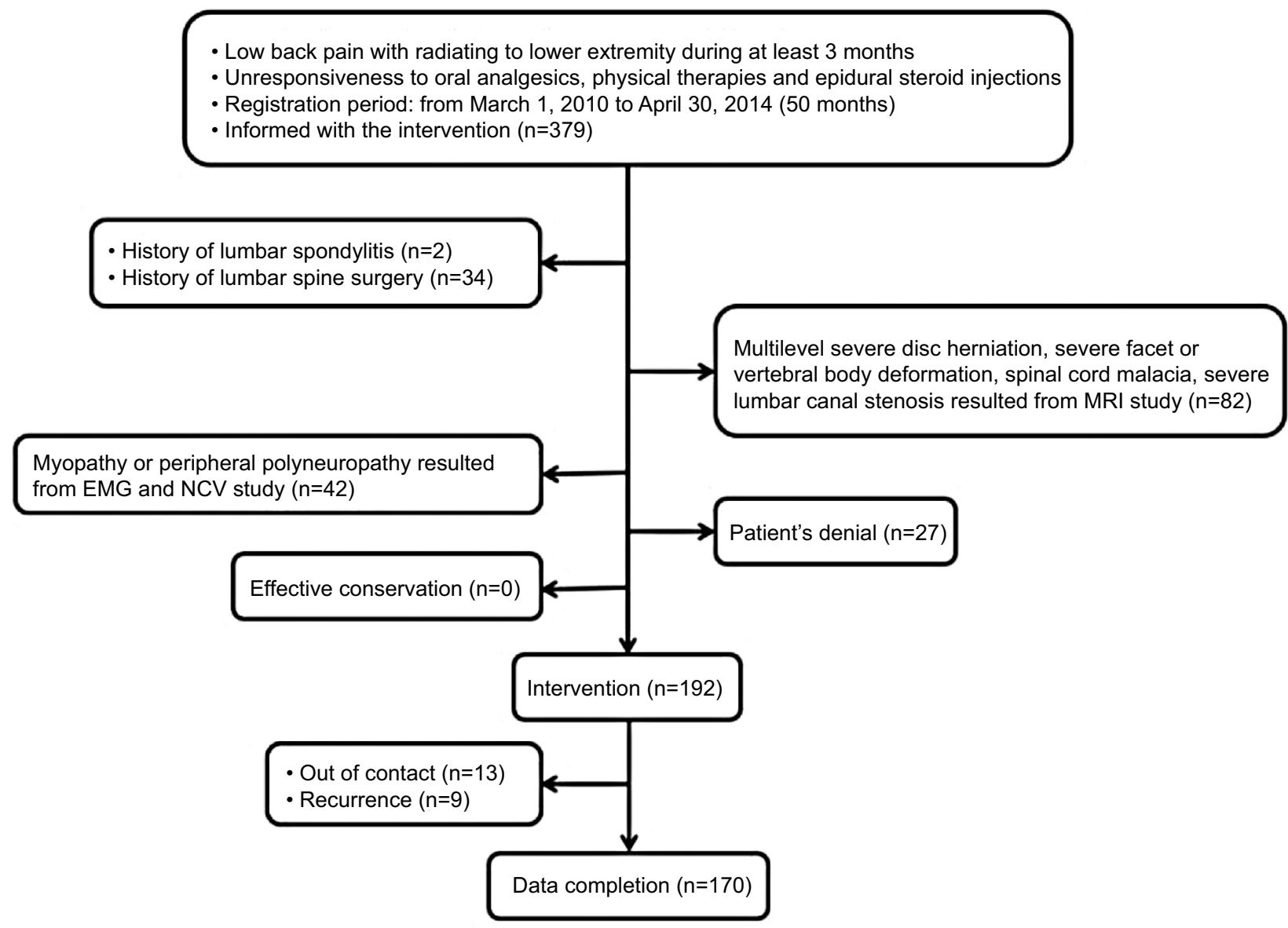

Figure 2 Subject enrollment.

Abbreviations: EMG, electromyography; NCV, nerve conduction velocity.

Table I Subjects baseline characteristics

\begin{tabular}{ll}
\hline Variables & Total $(\mathbf{n}=\mathbf{l 7 0})$ \\
\hline Sex & \\
M:F & $98: 72(\mathrm{M}, 57.6 \%)$ \\
Age (year) & \\
Mean \pm SD & $42.9 \pm 14.6$ \\
Range & $20-79$ (median 42) \\
Duration of symptoms (month) & \\
Mean & $16.4 \pm$ I7.8 \\
Range & $6-72$ (median 6) \\
Levels of target disc (case) & \\
LI/2 & 1 \\
L2/3 & 1 \\
L3/4 & 10 \\
L4/5 & 86 \\
L5/SI & 72 \\
Pfirrmann grading of disc degeneration & \\
II & 4 \\
III & 71 \\
IV & 95 \\
Herniation type & \\
Protrusion & 24 \\
Extrusion & 146
\end{tabular}

Abbreviations: $M$, male; $F$, female.
Good to fair outcomes were observed in 133 patients $(78.3 \%)$ at the end of 2 years, with greater than $50 \%$ decreases from the initial VAS scores (Figure 5). The change of VAS was correlated with perceived symptom changes in back and leg pain, but the coefficients of correlation were relatively different between groups with greater than $50 \%$ improvement and less than 50\% improvement. (Table 2)

\section{Recurrence and complications}

Recurrent lumbar disc herniation was defined as disc herniation at the same level, regardless of ipsilateral or contralateral herniation, with a pain-free interval greater than 6 months. The strict definition of recurrent disc herniation is the presence of herniated disc material at the same level, ipsilateralor contralateral, in a patient who has experienced a pain-free interval of at least 6 months since surgery. ${ }^{12}$ Of the recruited 192 subjects, nine subjects (4.7\%) were confirmed to have recurrent herniation according to this definition. The timing of the development of recurrence was determined by interview, without performing any clinical tests. The median time 


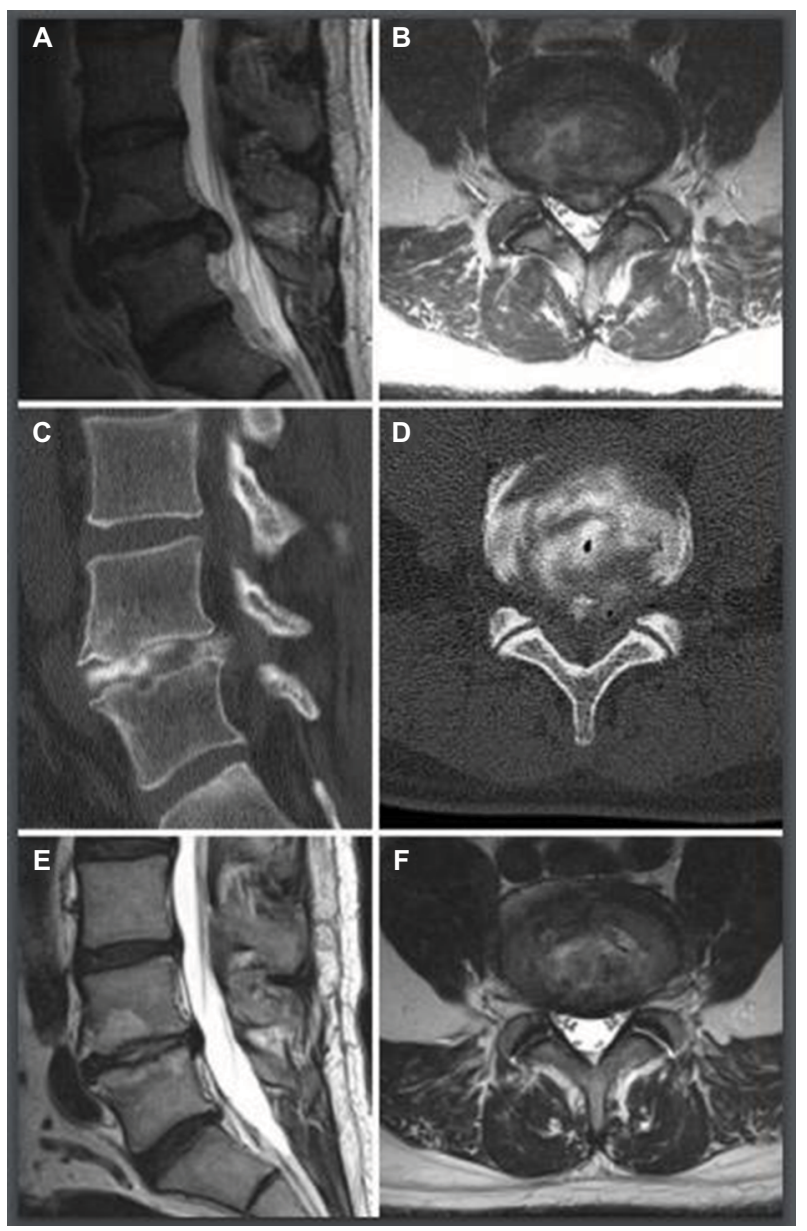

Figure 3 Example of MRIs and CT images in a subject.

Notes: MRIs and CT images of 43-year old male with L4/5 HNP. (A, B) Selective images of axial and sagittal MRls, pre-operative. (C, D) Selective CT images immediately after procedure, with intradiscal diffusion of contrast and post-ablation gas bubbles. (E, F) Selective images of axial and sagittal MRIs, post-operative I4 months.

Abbreviations: CT, computed tomography; HNP, herniated nucleus pulposus.

of recurrence was 8 months postoperatively, and the pain-free interval ranged from 7 to 23 months.

Three patients in the successful patient group experienced foot drop despite symptom improvement; immediately after the procedure, we classified the muscle power of their ankle dorsiflexors according to the grades of "trace" or "poor." Two patients improved to a normal power level within the first week, and the results of their electrophysiologic studies were within normal limits. However, one subject did not improve until 4 weeks postoperatively; on the basis of electrophysiologic study, we diagnosed this patient as having ipsilateral L5 radiculopathy with abnormal spontaneous activity. After conservative management over 6 months, the patient's foot dorsiflexor power improved to "fair" and at the 2-year survey, the power had increased to "good." The patient with this severe complication, a 69-year old male, had left L4/5 extruded disc in the subarticular region on MRI. In a retrospective analysis, the position of the tip in the fluoroscopic images was within the safe range: however, he mentioned experiencing an electric-like shock to the L5 dermatome, while simultaneously experiencing sudden involuntary lower limb contraction during the procedure.

We diagnosed another patient with regional cellulitis adjacent to the needle puncture site, but the symptoms and signs improved after the patient took oral antibiotics. Eleven patients complained of temporary focal pain at the needle puncture site during trunk motion, which we believed was muscle soreness, but the pain showed complete resolution within 4 weeks.

\section{Discussion}

Lumbar spinal pain leads to impaired locomotion and limited participation in activities. In particular, disc disease in the industrialized population is very common, and the resulting limitations and work interruptions lead not only to increased medical costs for treatment but also decreased productivity. In light of this, the optimal treatment of patients with HNP includes a short treatment time, curative effects, rapid return to daily life, and cost-effectiveness. Minimally invasive interventional procedures and devices have been proposed to meet this need, and tissue ablation using catheter insertion has been shown to decrease local pressure and cauterize abnormally sprouting nerve endings. ${ }^{13}$

Annulus fibrosus tear is an important factor in disc disorder, and the biochemical and neuralgic reactions of this lesion are closely related to the symptoms. ${ }^{14,15}$ Annulus fibrosus tear begins within the intervertebral disc, and as it progresses to the outside, the probability of nucleus pulposus herniation increases. The epidural and foraminal space-occupying tissues and inflammation around the torn or irritated lesion may be targets for noninvasive spinal interventions. The navigable catheter we use in our approach was designed to reach the torn annulus from within the disc and to reduce intradiscal pressure by coblating the herniated disc.

We were very cautious in selecting the subjects for this approach. In general, if symptoms persist for more than 6 months and the response to conservative treatment is insignificant, surgical treatment is recommended. ${ }^{3}$ In this study, patients with more than 6 months of symptom duration were recruited and treated with conservative treatment for at least 3 months. Our surgical procedure was only performed on subjects who were unresponsive to conservative treatment and therefore not expected to have a good prognosis without further intervention. 


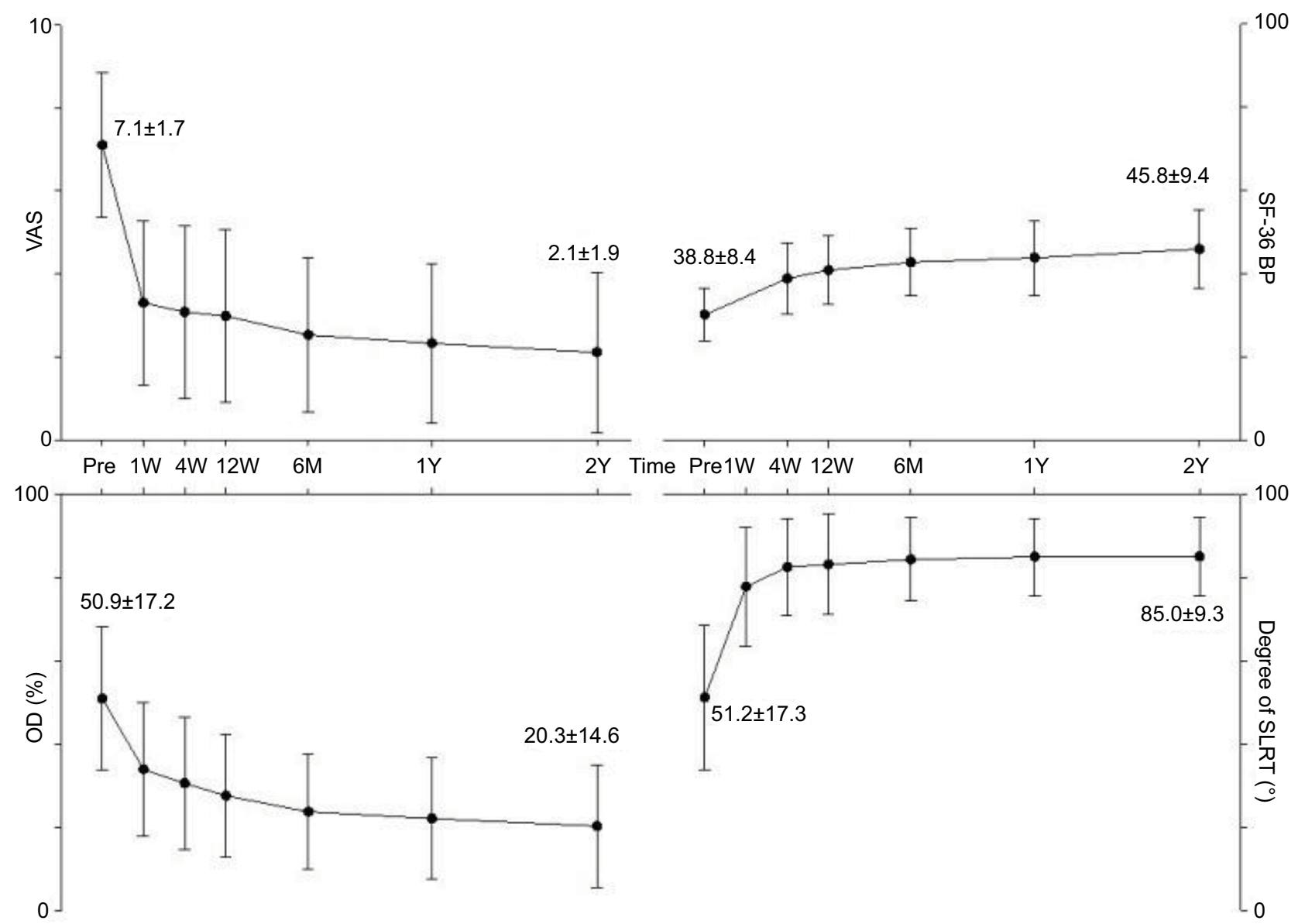

Figure 4 Results of clinical outcomes.

Abbreviations: Pre, pre-procedural; W, week; Y, year; VAS, visual analog scale; SF-36 BP, short form-36 of bodily pain scale; SLRT, passive straight leg raise test.

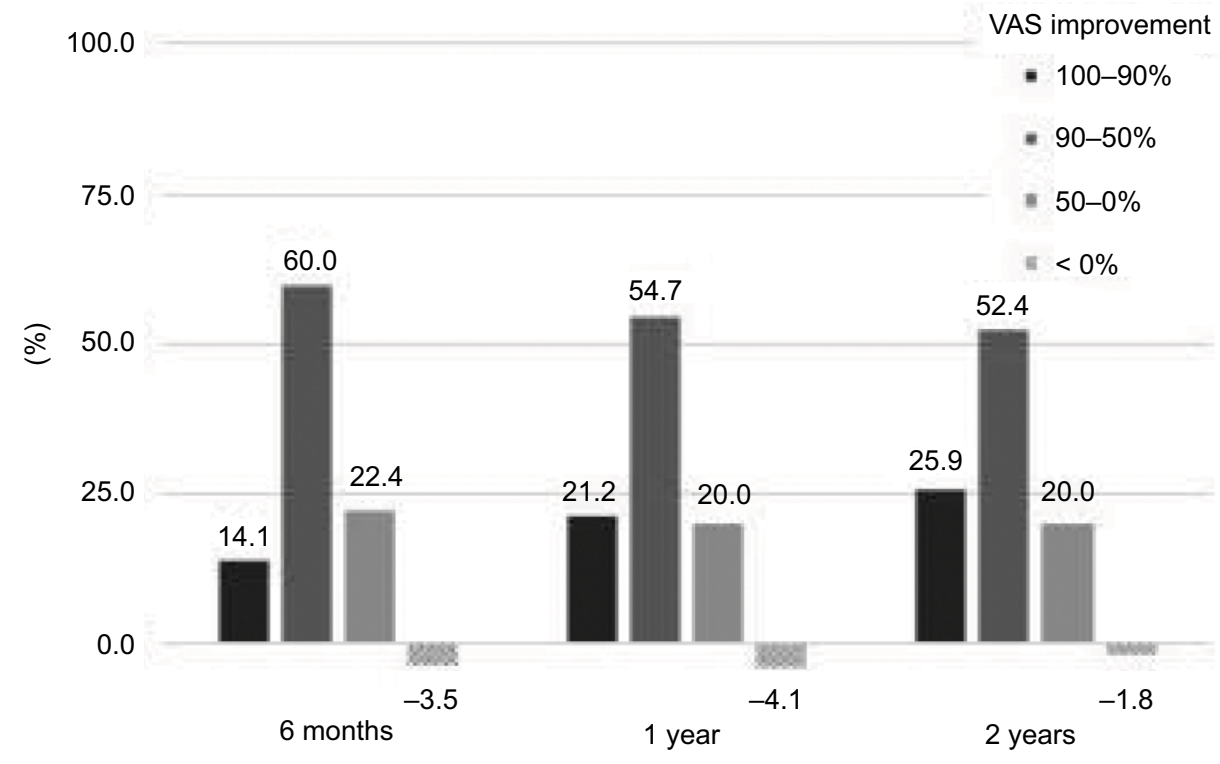

Figure 5 Clinical efficacy of the procedure and device.

Abbreviation: VAS, visual analog scale. 
Table 2 Correlation with perceived pain improvement of back and leg

\begin{tabular}{ll}
\hline Perceived change & Change of VAS during 2 years \\
\hline Total & \\
Back & 0.74 \\
Leg & 0.77 \\
Group with $>50 \%$ improvement & \\
Back & 0.46 \\
Leg & 0.54 \\
Group with $<50 \%$ improvement & \\
Back & 0.76 \\
Leg & 0.37 \\
\hline
\end{tabular}

Notes: Perceived change of symptoms has been surveyed by 5 -point Likert scale. The correlation (Spearman's rho) was significant with $P<0.05$ in all instances. Abbreviation: VAS, visual analog scale.

Notably, in this study, we found that clinically significant decompression is possible by removing very little herniated tissue. Surgery using a radiofrequency generator lasts for approximately 300 seconds during which $1.35 \mathrm{~g}$ of nuclei is removed even under laboratory conditions, ${ }^{16}$ this decreases by approximately half in vivo, to below $0.5 \mathrm{~cm}^{3}$. This is comparable to the $2.0 \mathrm{~cm}^{3}$, at which a significant amount of nuclei is removed and grossly confirmed in cases of open discectomy. ${ }^{17-19}$ However, a 2-year long-term outcome study of lumbar discectomy showed that the degree of disc removal did not influence outcomes or the complication rate. ${ }^{19}$

Although the far-lateral approach is used to minimize the possibility of damage to the spinal nerves, this method is challenging when approaching discs at L5/S1 and contact with the spinal nerves or dural sleeves may occasionally occur. In this study, injury to the L5 spinal nerve did occur, and the clinical presentation comprised mainly ankle or big toe extensor weakness as opposed to sensory abnormalities of the dermatome. Three of 170 patients experienced ankle and big toe extensor weakness and two had L5/S1 lesions: for the latter two patients, electrophysiologic study revealed no evidence of radiculopathy, and full motor recovery occurred within 4 weeks. These conditions may have been caused by a nerve conduction block. ${ }^{20}$

One patient experienced a severe foot drop caused by actual L5 root injury, which was confirmed as a significant axonal injury on electrophysiologic study. We assume that this occurred in contact with the spinal root during tip ablation, possibly because of electrical shock, thermal damage, or mechanical stimulation. It is important to note that although the position of the tip does not cross the annular outer membrane in our procedure protocol, the outer membrane did not function as a barrier in this case. Surgeons should proceed with this procedure cautiously when the patient reports a radicular sensation with weak and short electrical stimulation before ablation in order to contact with neural tissues.

The herniation recurrence rate was around $4.7 \%$ based on the totally collected data, which is similar to the reported recurrence rate after discectomy. Several studies have reported lumbar disc herniation recurrence rates of 5\%-12\%. ${ }^{21-28}$ Thirteen of the 192 patients dropped out of contact after hospital discharge. According to our data, assuming all of them had recurred or not, the recurrence rate was $4.7 \%-11.5 \%$ during the 2-year period following the intervention.

The reason why we evaluated the perceived symptom of subjects in back and leg pain at the 2-year follow-up visit was that the back pain of most subjects who had less improvement in the preliminary study was relatively prominent. First, the perceived changes of both back and leg pain were revealed a high correlation with VAS change after 2 years. However, according to the criteria of VAS 50\% improvement, the group with more than $50 \%$ improvement (from 7.3 to 1.3 in VAS on average, $n=133$ ) showed similar coefficients of correlation in leg and back pain, while the group with less than 50\% improvement (from 6.3 to 4.9 in VAS on average, $n=37$ ) showed lower correlation of leg pain and higher correlation of back pain. As we suspected in our preliminary findings, if the clinically significant improvement did not occur after this procedure, we can assume that the back pain was complained more than leg pain in initial evaluation. It is difficult to interpret of residual back pain after the procedure with HNP pathology as it is based on the observation and analysis, the unresponsive back pain after the procedure can be regarded not as a sequela of HNP but as other disc disorder with a different pathology. Epidural fibrosis may be a contributing factor to post-surgical persistent pain in approximately $30 \%$ of patients with failed back surgery syndrome. ${ }^{29,30}$ But, the catheter of this device does not work within epidural space. Myofascial pain due to paraspinal musculature injury can be suspected. ${ }^{31}$ Unilateral insertion of the $1.9 \mathrm{~mm}$ diameter needle may damage slightly the muscle and fascia tissues, but it is unlikely that this damage can be represented by persistent axial low back pain. Secondary instability to alter the distribution of spinal load can be a contributing factor. Discectomy may result in changes to the biomechanic balance in the spinal column, and the intradiscal load redistribution provides a possibility to accelerate preexisting disc degeneration. ${ }^{32,33}$ Further studies are suggested about the degree of instability and degeneration relevant with removal amount, and feasible rehabilitation strategies.

This study has some limitations. The background of the development of this device was oriented toward the goals of minimal invasiveness, rapid resumption of work, and 
significant clinical effects. However, as we did not quantify the functional improvement for return to work and daily life after discharge, we could only indirectly deduce them according to the measurable symptom and pain-related functional recovery. Furthermore, our failure to track 13 subjects may have had a significant effect on the results. Given that all the 13 subjects experienced recurrence, this can be interpreted as occurring either due to symptoms recurring or to unsuccessful treatments, and some unexpected complications were missed in the reporting of the results.

\section{Conclusion}

The general treatment goals of spinal surgery in patients with lumbar disc herniation include rapid symptom improvement, early return to work, and minimization of complications and recurrence. The radiofrequency focal ablation technique using a navigable plasma disc decompression catheter is a viable and optimized option for the minimally invasive treatment of lumbar disc herniation, although critical attention should be given to the possibility of electrical damage to neural tissue. However, further technological advancements are necessary to improve the efficiency of tissue removal and resolve safety issues.

\section{Acknowledgments}

This research was supported by a grant of the Korean Health technology R\&D Project through the Korea Health Industry Development Institute (KHIDI), funded by the Ministry of Health \& Welfare, Republic of Korea (grant number: HI14C3477).

\section{Author contributions}

NH Kim: Study design, management, data collection, statistical analysis, writing up of the first draft of the paper. YK Hong: study design, revision of the manuscript. SH Lee: study design, data collection, critical revision of the article for intellectual content. All authors contributed toward data analysis, drafting and revising the paper and agree to be accountable for all aspects of the study.

\section{Disclosure}

The author Sang-Heon Lee invented the device and intervention method and has some royalty interest in the device. The other authors report no conflicts of interest in this work.

\section{References}

1. Kahanovitz N, Viola K, Muculloch J. Limited surgical discectomy and microdiscectomy. A clinical comparison. Spine. 1989;14(1):79-81.

2. Spengler DM. Lumbar discectomy. Results with limited disc excision and selective foraminotomy. Spine. 1982;7(6):604-607.
3. Blamoutier A. Surgical discectomy for lumbar disc herniation: surgical techniques. Orthop Traumatol Surg Res. 2013;99(1 Suppl.):S187-S196.

4. Yoon SJ, Choi KO, Yoon SM, et al. A statistical analysis of the causes of cancellation of elective operation. Korean JAnesthesiol. 2001;40(2): 139-143.

5. Hong YK, Derby R, Wolfer LR, et al. An assessment of a new navigatable percutaneous disc decompression device (l'DISQ) through histologic evaluation and thermo-mapping in human cadaveric discs. Pain Med. 2012;13(8):1000-1003.

6. Lee SH, Derby R, Sul D, et al. Efficacy of a new navigable percutaneous disc decompression device (L'DISQ) in patients with herniated nucleus pulposus related to radicular pain. Pain Med. 2011;12(3):370-376.

7. Jarvik JG, Hollingworth W, Martin B, et al. Rapid magnetic resonance imaging vs radiographs for patients with low back pain: a randomized controlled trial. JAMA. 2003;289(21):2810-2818.

8. Rabin A, Gerszten PC, Karausky P, et al. The sensitivity of the seated straight-leg raise test compared with the supine straight-leg raise test in patients presenting with magnetic resonance imaging evidence of lumbar nerve root compression. Arch Phys Med Rehabil. 2007;88(7):840-843.

9. Pfirrmann CW, Metzdorf A, Zanetti M, Hodler J, Boos N. Magnetic resonance classification of lumbar intervertebral disc degeneration. Spine. 2001;26(17):1873-1878.

10. Bogduk N, editor. Practice Guidelines for Spinal Diagnostic and Treatment Procedures. San Francisco, CA: International Spine Intervention Society; 2013.

11. Fardon DF, Milette PC, Combined Task Forces of the North American Spine Society, American Society of Spine Radiology, and American Society of Neuroradiology. Nomenclature and classification of lumbar disc pathology. Recommendations of the Combined Task Forces of the North American Spine Society, American Society of Spine Radiology, and American Society of Neuroradiology. Spine. 2001;26(5):E93-E113.

12. Swartz KR, Trost GR. Recurrent lumbar disc herniation. Neurosurg Focus. 2003;15(3):E10-4.

13. Gangi A, Basile A, Buy X, Alizadeh H, Sauer B, Bierry G. Radiofrequency and laser ablation of spinal lesions. Semin Ultrasound CT MR. 2005;26(2):89-97.

14. Peng B, Hao J, Hou S, et al. Possible pathogenesis of painful intervertebral disc degeneration. Spine. 2006;31(5):560-566.

15. Peng B, Wu W, Hou S, et al. The pathogenesis of discogenic low back pain. J Bone Joint Surg Br. 2005;87(1):62-67.

16. Lee S-H, Kim NH, Kim G-H, et al. Characteristics of molybdenum as a plasma-generating electrode. Sci Adv Mater. 2016;8(9):1844-1847.

17. McGirt MJ, Eustacchio S, Varga P, et al. A prospective cohort study of close interval computed tomography and magnetic resonance imaging after primary lumbar discectomy: factors associated with recurrent disc herniation and disc height loss. Spine. 2009;34(19):2044-2051.

18. Schmid G, Witteler A, Willburger R, Kuhnen C, Jergas M, Koester O. Lumbar disk herniation: correlation of histologic findings with marrow signal intensity changes in vertebral endplates at MR imaging. Radiology. 2004;231(2):352-358.

19. Fountas KN, Kapsalaki EZ, Feltes CH, et al. Correlation of the amount of disc removed in a lumbar microdiscectomy with long-term outcome. Spine. 2004;29(22):2521-2524.

20. Zhang X, Roppolo JR, de Groat WC, Tai C. Mechanism of nerve conduction block induced by high-frequency biphasic electrical currents. IEEE Trans Biomed Eng. 2006;53(12 Pt 1):2445-2454.

21. Davis RA. A long-term outcome analysis of 984 surgically treated herniated lumbar discs. J Neurosurg. 1994;80(3):415-421.

22. Findlay GF, Hall BI, Musa BS, Oliveira MD, Fear SC. A 10-year followup of the outcome of lumbar microdiscectomy. Spine. 1998;23(10): $1168-1171$.

23. Loupasis GA, Stamos K, Katonis PG, Sapkas G, Korres DS, Hartofilakidis G. Seven- to 20-year outcome of lumbar discectomy. Spine. 1999;24(22):2313-2317.

24. Moore AJ, Chilton JD, Uttley D. Long-term results of microlumbar discectomy. Br J Neurosurg. 1994;8(3):319-326. 
25. Ruetten S, Komp M, Godolias G. An extreme lateral access for the surgery of lumbar disc herniations inside the spinal canal using the full-endoscopic uniportal transforaminal approach-technique and prospective results of 463 patients. Spine. 2005;30(22):2570-2578.

26. Weiner BK, Dabbah M. Lateral lumbar disc herniations treated with a paraspinal approach: an independent assessment of longer-term outcomes. J Spinal Disord Tech. 2005;18(6):519-521.

27. Yeung AT, Tsou PM. Posterolateral endoscopic excision for lumbar disc herniation: Surgical technique, outcome, and complications in 307 consecutive cases. Spine. 2002;27(7):722-731.

28. Yorimitsu E, Chiba K, Toyama Y, Hirabayashi K. Long-term outcomes of standard discectomy for lumbar disc herniation: a follow-up study of more than 10 years. Spine. 2001;26(6):652-657.
29. Chopra P, Smith HS, Deer TR, Bowman RC. Role of adhesiolysis in the management of chronic spinal pain: a systematic review of effectiveness and complications. Pain Physician. 2005;8(1):87-100.

30. Trescot AM, Chopra P, Abdi S, Datta S, Schultz DM. Systematic review of effectiveness and complications of adhesiolysis in the management of chronic spinal pain: an update. Pain Physician. 2007;10(1):129-146.

31. Kawaguchi Y, Matsui H, Tsuji H. Back muscle injury after posterior lumbar spine surgery. A histologic and enzymatic analysis. Spine. 1996;21(8): 941-944.

32. Onesti ST. Failed back syndrome. Neurologist. 2004;10(5):259-264.

33. Kumar MN, Baklanov A, Chopin D. Correlation between sagittal plane changes and adjacent segment degeneration following lumbar spine fusion. Eur Spine J. 2001;10(4):314-319.

\section{Journal of Pain Research}

\section{Publish your work in this journal}

The Journal of Pain Research is an international, peer reviewed, open access, online journal that welcomes laboratory and clinical findings in the fields of pain research and the prevention and management of pain. Original research, reviews, symposium reports, hypothesis formation and commentaries are all considered for publication

\section{Dovepress}

The manuscript management system is completely online and includes a very quick and fair peer-review system, which is all easy to use. Visit http://www.dovepress.com/testimonials.php to read real quotes from published authors. 